\title{
Fuel Station Monitoring and Automation based on WSN
}

\author{
Ehab AbdulRazzaq Hussein, Mahran Obaid Waheed
}

Department of Electrical, Faculty of Engineering, Babylon University, Iraq

\begin{tabular}{l} 
Article Info \\
\hline Article history: \\
Received Feb 7, 2018 \\
Revised May 10, 2018 \\
Accepted May 16, 2018 \\
\hline
\end{tabular}

\section{Keyword:}

Automation

Fire protection

Fuel station

LABVIEW

Monitoring

NI WSN

Temperature protection

\begin{abstract}
The Iraqi fuel station still now uses old technologies to control its activities from filling tanks to the filling cars. Automate the activity of fuel station is the objective of this work. The aims of fuel station automation are to save the fuel quantities and qualities supplied in fuel station, and to keep the fuel station, the worker and its main parts safe. This work uses the national instrument wireless sensor network (NI WSN). The NI WSN used to automate the protection system and level controlling system which makes the fuel station work under normal ambient temperature, and normal protection conditions. Automation based on a wireless sensor network gives excellent capabilities to automate and monitor fuel station. Through the user interface window the user monitor the status of actuators, protection system controller messages, fuel levels, water level, environment temperature, power source and its quality. The soft controller developed was built within The LABVIEW environment. The results of controller give the desired action through "on" and "off" states of the actuators
\end{abstract}

Copyright $\odot 2018$ Institute of Advanced Engineering and Science. All rights reserved.

\section{Corresponding Author:}

Ehab Abdul Razzaq Hussein,

Department of Electrical Engineering,

Faculty of Engineering,

Babylon University, Babylon, Iraq.

Email: dr.ehab@itnet.uobabylon.edu.iq

\section{INTRODUCTION}

Automation and monitoring have a big role in all life sides, industrial fields an example. One of the main tools of automation is a wireless sensor network (WSN), its use a group of sensors connected with each other via a wireless network to ensure connectivity between any sensor in industrial location and the main station, this makes data on unsafe location easily arrive to the main station. Automation based on a wireless sensor network gives excellent capabilities to automate and monitor fuel station, especially it was an industrial location which contains the toxic and flammable gases. LABVIEW is industrial software that have good user interface compare with other software, it used to implement the processing of wireless sensor network data to control the actuators in the fuel station. Figure 1 shows the simplified block diagram of the system done in this work. Figure 1 shows that there are sensors as input to the system and outs of the system used to control the actuators through wire across the microcontrollers or wireless through the WSN end nodes. The sensors that used are temperature, smoke, level, water, light, voltage, phase failure, pressure as inputs to the system. The actuators are submersible motor, dispenser motor, filing motor, cooling motor, alarm buzzer, lights, engine ignition and starter of the local source generator. 


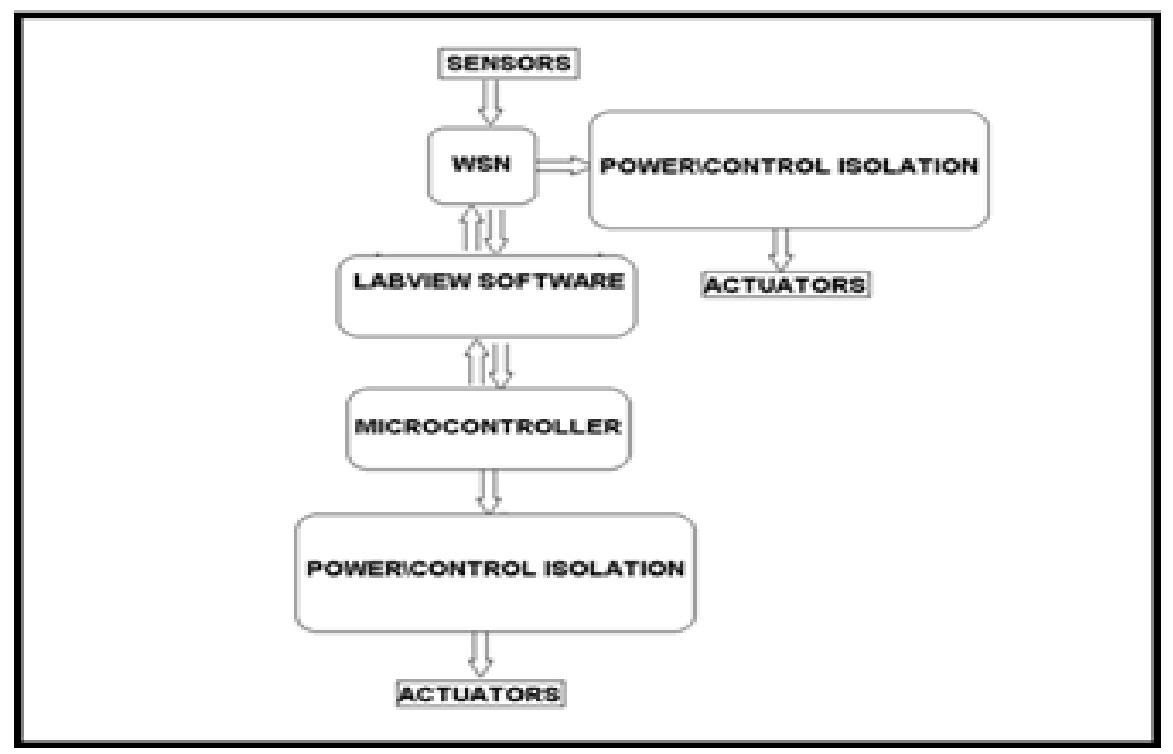

Figure 1. Blcok diagram for gureproposed system

\section{RELATED WORK}

The survey covers two topics. The first includes the use of the wireless sensor network in automation and industrial applications. The second is the using of the national instrument software and hardware to implementation the automaton based on NI WSN.

There are recent efforts that deal with using wireless Sensor Network in industrial application. The interesting sides were advantages of wireless technologies beyond the wire technologies, where:

a. Waqas Ikram \& Nina F. Thornhill, 2010 [1] presents the problems of wired technologies in industrial applications and the benefits of technologies that use wireless communication. In the study, the researcher concludes that low power and short range wireless communication are suitable for industrial automation applications.

b. Gang Zhao \& Davis, 2011 [2] show that the wireless sensor network bring several advantages over wired technologies for monitoring and control of industrial systems, in the study, the researcher present the challenges that face the transition from wired to wireless as power management, smart routing and self healing.

c. Dr. P.Velmani, 2013 [3] demonstrates the possibility to apply the wireless sensor network in industrial applications by use of a small detector system, stratified Analyzer to a micro electro mechanical system and data acquisition of machine vision. Also the author showed that there are two types of WSN use in industrial fields, the first is the intelligent management of industry and the second is the secure management of industries. Where machine vision system, smart transmitter, process monitoring and building automation are classified as intelligent industrial management and fire alarm, leak detection, unauthorized vehicle detection and smoke detection are classified as secure industrial management. Also the author shows the challenges that face migrating from wired to WSN technologies are coverage area, the signal strength, balance between battery life and rate of data update, WSN protocols, wireless security and quality of service requirements.

d. A. Ajith Kumar S., Knut $\varnothing$ vsthus, Lars M. Kristensen, 2014 [4] show that there are differences between the conventional WSN and industrial WSN (IWSN) where industrial application need specific protocols based on certain factors. The authors refer to main factor must be not change when replace the wired with IWSN technologies, quality of service (Qos) is the main factor, at same time low cost low power IWSN. According to the author there are six classes of the industrial system, they are safety system, close loop regulatory system, close loop supervisory system, open loop control system, alerting system and information gathering system. According to these class requirements the protocols of medium access control, transport and routing function will be selected.

National instruments introduce LABVIEW software and its supported hardware as NI WSN and data acquisitions (DAC). There are good works to implement automation and industrial application by LABVIEW software and NI products, as bellow: 
e. Basil Hamed, 2012 [5] presents the automation system for smart house using LABVIEW software as a main controller, although there are sub controllers locally remote controlled and globally through internet controller. The control system can monitor the temperature, lighting, humidity, gas density, fire and burglar security alarms. The system consists of five subsystems, security, lighting, remote control, temperature and main house power subsystems. There are two interfaces, computer and remote controller interface.

f. S. Rajesh Kumar \& S. Rameshkumar, 2013 [6] propose control and monitoring system using data acquisition and LABVIEW. Temperature sensor with DAQ and LABVIEW software used to implement the control system. Author concludes that the use of Ethernet in industrial automation is a good solution because of speed of Ethernet that reach up Gbps.

g. S. Sathiysmoorthy, 2014 [7] shows that the machine vision offers the best solution for quality control in industrial applications. Dimensional measurements, process control of the image processing and optical character recognition (OCR) techniques are used to checking products parts in finishing stage of the production line. Machine Vision (MV) approach provides the automatic inspection based on the image processing. MV has three parts image acquisition, image processing and controller decision. The optical gauging of shaft, engine assembly and inspection of level and cap of bottle are machine vision applications presented in the work. Author concludes that the using of MV in industrial applications reduce the cost and subsequent stages of production and improve the quality of products.

h. Poornendu K, Manoj G \& Kannan E. P., 2015 [8] present monitoring and controlling thermal power plant parameters such as pressure, humidity, and temperature. The two parts of the system are hardware and software. The hardware part consists of wireless transceiver and microcontroller, but the software part built within LABVIEW where serial port used in virtual instrument system architecture (VISA). The authors conclude that the system gives efficient results in monitoring and data acquisition.

i. A. Dhadiwiwal, Payal Disoja \& Prof Amit H. Palve, 2015 [9] show that the smart power control in industrials, large and small buildings are the best solution to power loss due to the working of unnecessary loads, because of the state monitoring of electrical devices in the building using WSN technology. The system implemented by the microcontroller which led to flexible, low cost and robust system.

\section{THE PROPOSED SYSTEM}

Figure 2 shows the main component of a fuel station and its equipments. The fuel station is the industrial location where fuel is dispensed into the fuel tank of cars or fuel containers. Fuel station consists of the following components, storage tank, fuel dispenser, submersible pump, filling pump, and filling system.

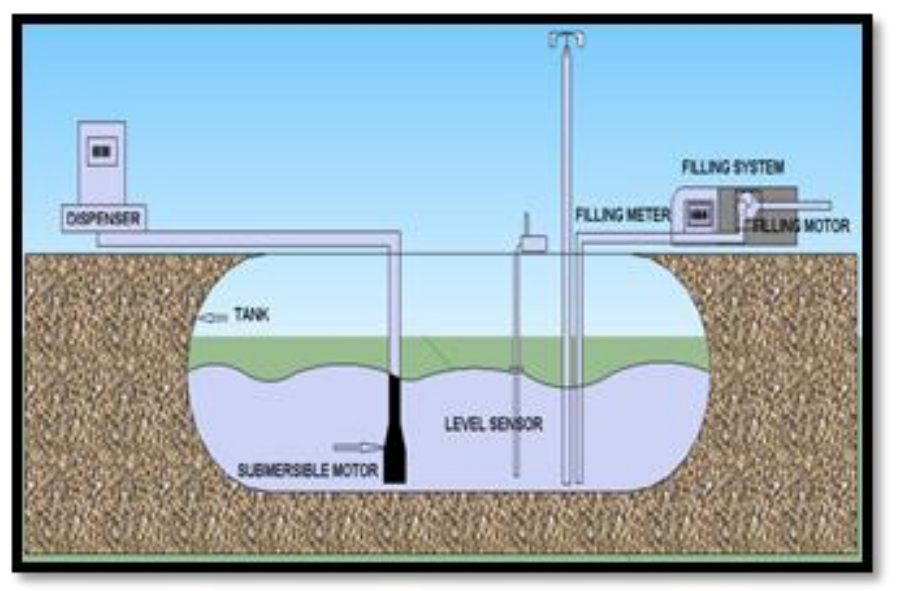

Figure 2. Fuel station components

The main goal of the proposed system is to design a fuel station controller to automate and monitor the main activities of fuel station. The main activities in the fuel station which deal with in researcher work are, main tank and car tank filing system automation, fire protection system, and temperature protection system, internal and external lighting system. The tools that used in this work are NI WSN system, laptop, LABVIEW software, microcontroller, temperature sensor, smoke sensor, water detector, level sensor 
emulator, density sensor emulator, globallocal power source emulator, and photo sensor. The NI WSN system contains gateway node, end node, and routing node which forms the WSN system. The WSN system is responsible to receive sensor data from sensors and transmit commands to the actuators and to the main station (Laptop). The laptop used as hardware that responsible to execute the main soft controller. The main controller was being built by the LABVIEW software. The microcontroller is used to implement the main system without WSN as pre model and used as assistance with the WSN to read information and transfer command to the main controller. Temperature and smoke sensors used to give information about the fuel station environment to decide if the state is safe or unsafe. The level sensor emulator and the density sensor emulator used to give information for the main controller to control operation of submersible pumps, fuel dispenser, and the main tank filling system. The globallocal power source emulator used to emulate the working of main power supply (global source) and local power supply (power generator set). The mission of globalllocal power source emulator is to give information for the automatic power changer system to decide which power is appear and provide the proper commands. Photo sensor used to provide information about light intensity for the main controller to control the lighting system internal and external locations of the fuel station. Figure 3 is briefly illustrating to the proposed system where the heart of system is the main controller (Laptop).The main controller connected to the sensors and the actuators wirelessly through the WSN and connected to sub controllers logically (through the LABVIEW software). The remote actuators connected to NI WSN end nodes via power Isolators as photo couplers or relays. The local actuators connected to the local sub controller via USB cable and power insulator. Where the elements of the Figure 3 defined by the Table 1.

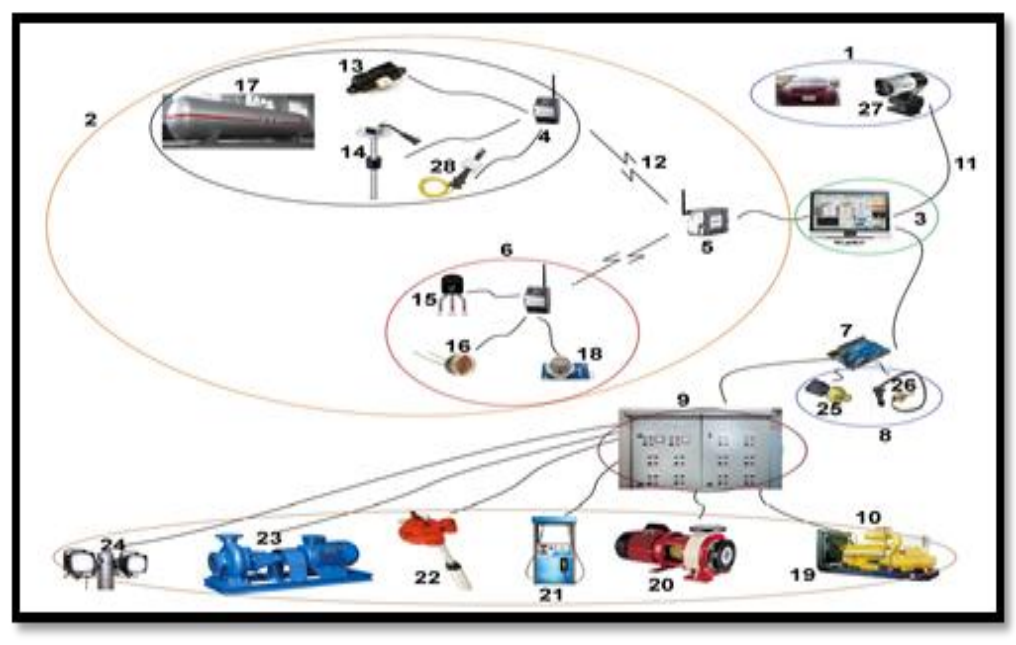

Figure 3. Proposed system implementation

Table 1. The Definitions of the Proposed System Elements

\begin{tabular}{clcl}
\hline No. & \multicolumn{1}{c}{ Component } & No. & \multicolumn{1}{c}{ Component } \\
\hline 1 & ANPR system (beyond the paper scope) & 15 & Temperature sensor \\
2 & WSN components & 16 & Light sensor \\
3 & PAC "programmable automation controller" & 17 & Main fuel tank \\
4 & WSN end node & 18 & Smoke sensor \\
5 & WSN gateway & 19 & Generator sets \\
6 & Environmental sensors & 20 & Water pump \\
7 & Microcontroller (Arduino) & 21 & Fuel dispenser \\
8 & Generator sets, sensors & 22 & Submersible motor \\
9 & Power distribution panel & 23 & Filling motor \\
10 & Fuel station equipments & 24 & Lighting sources \\
11 & Wire connection & 25 & Engine oil Pressure sensor \\
12 & Wireless connection & 26 & Engine temperature sensor \\
13 & Distance sensor (level sensor) & 27 & Camera \\
14 & Density sensor & 28 & Water sensor \\
\hline
\end{tabular}

The systems that contained within the proposed system are fire protection system, high temperature protection system, automatic power changer system, level controller system, and lighting controller system 
which implemented as:

\subsection{Fire protection system}

Figure 4 shows that the user interface of the fire protection system. The system inputs are type of protection, temperature threshold value, temperature sensor value, smoke sensor, and jump fire alarm. The output of the fire protection system is the fire alarm. The first case of fire detected (fire alarm $=$ on) and the second case is no fire detected (fire alarm=off) or normal state. The sensors which used are LM35 as the temperature sensor and MQ-2 as smoke sensor. LM35 is the analogue sensor has the data sheet in reference [10] and MQ-2 used as digital smoke sensor which gives an indicator under specific conditions, according to the factory settings, its data sheet in reference [11]. The LABVIEW code that achieves the fire protection system shown in the Figure 5.

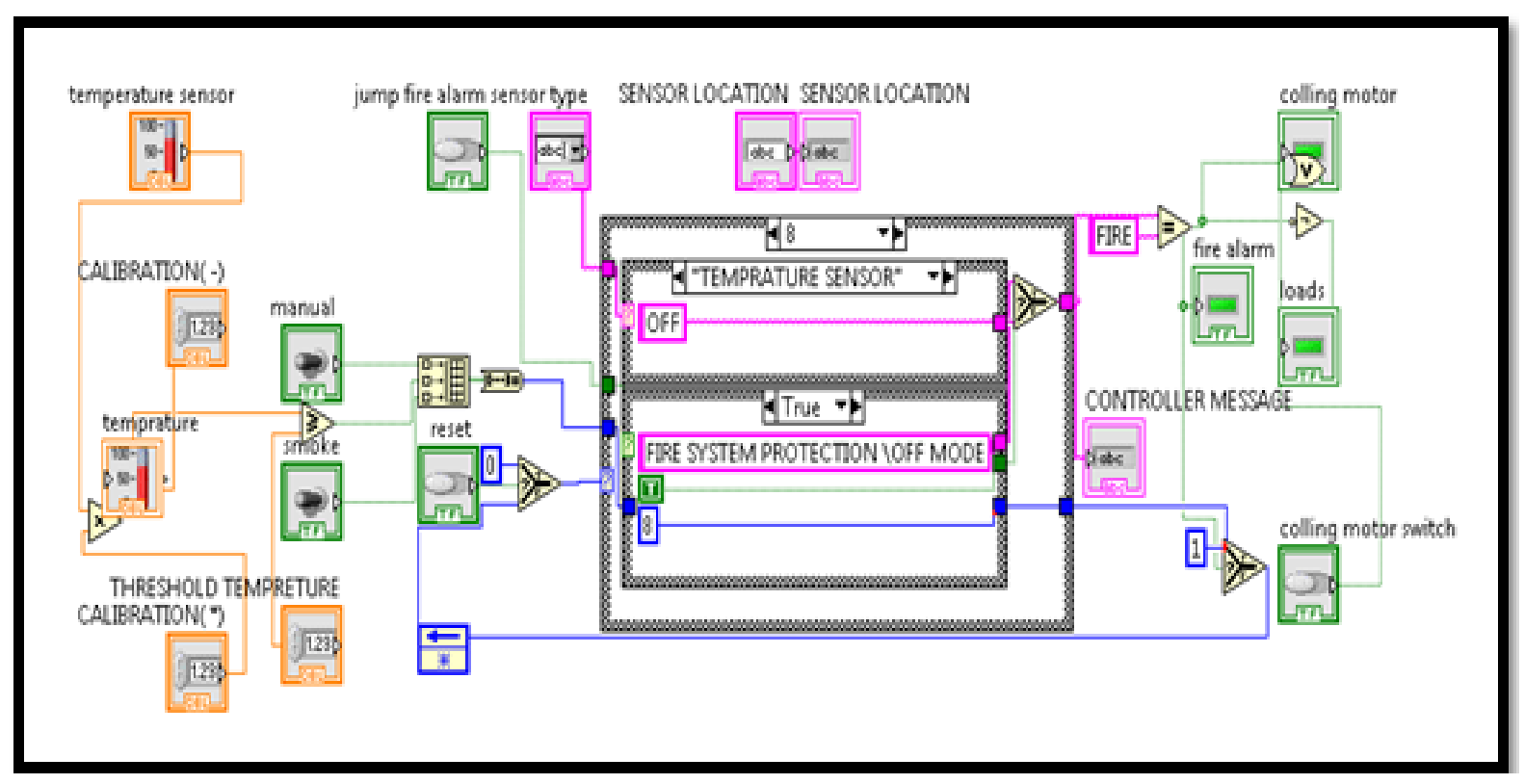

Figure 4. User interface of fire protection system

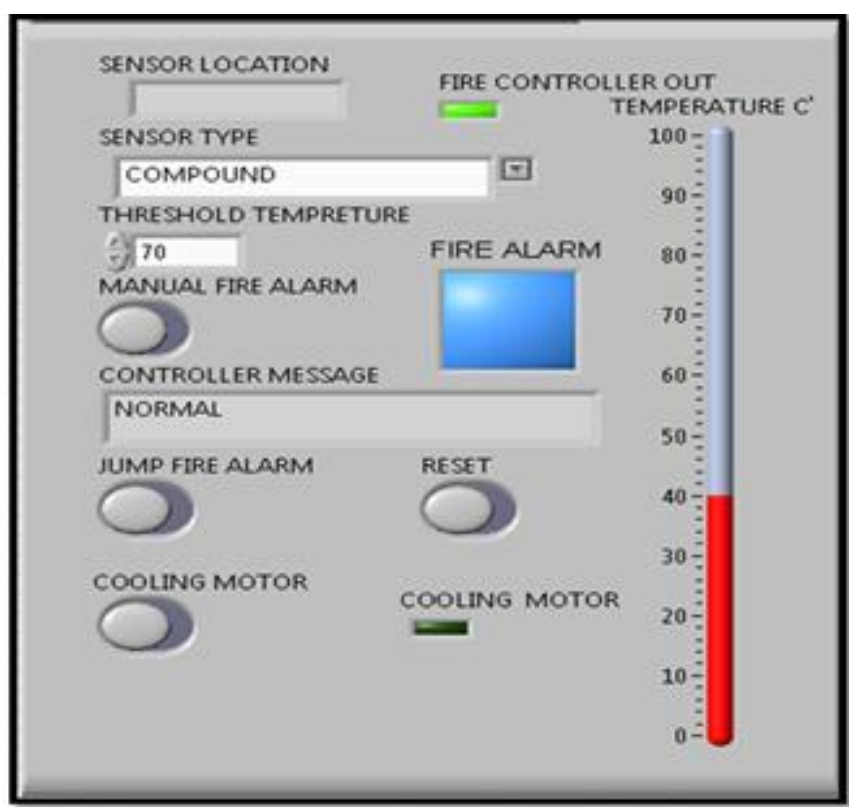

Figure 5. Fire protection system code 


\subsection{High temperature protection system}

The nature of fuel station makes the working equipments (dispensers, filling system...) in direct contact with the outer environment factors as ambient temperature which increases in the summer season in Iraq. Also the probability of an accident is directly proportional to increase the temperature above the normal state in the flammable environment. High temperature protection system was introduced to protect these equipments against an abnormal increase in the ambient temperature, The temperature controller uses simple techniques to overcome the oscillation phenomena which make the system unstable. This technique clearly explained in the Figure 6 where the output may be on or off for the specific overlaps interval $\left(R_{2}\right.$ and $\left.R_{4}\right)$ which lie between the minimums and maximums allowable intervals for upper and lower levels. Without this technique, the system oscillates between switching on and off of the loads if the sensor data swing about the threshold value. The user interface of the high temperature subsystem is shown in the Figure 7.

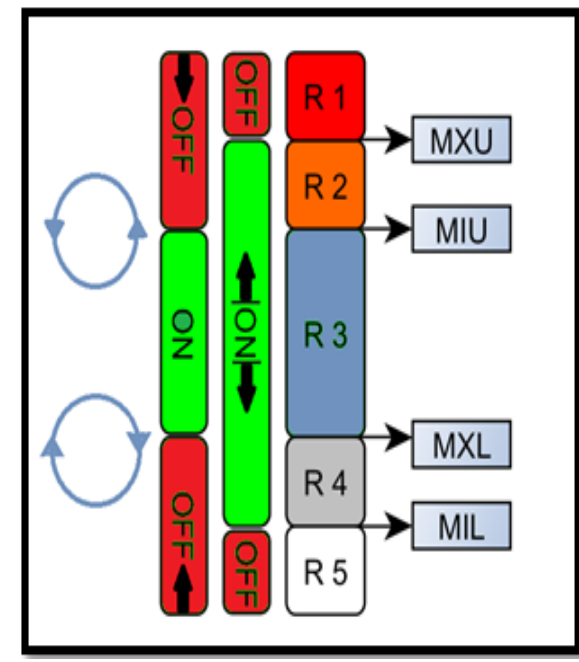

Figure 6. Oscillation prevention technique

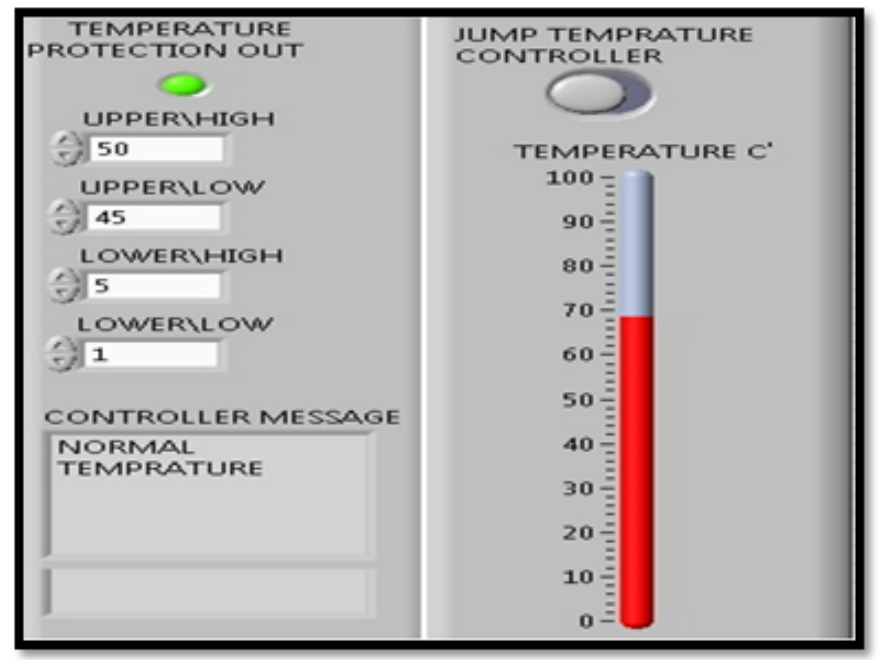

Figure 7. User interface of high temperature protection system

\subsection{Automatic power changer system}

Unfortunately the general power source in Iraq has problems making the local power source necessary to make the fuel station services available along the working time. The automatic power changer system used to control the activities of changing, checking the power state, and checking the health of the generator set. The system tested by using the emulator card that's designed to emulate the behavior of the power sources (global and local power sources). Figure 8 shows the controller user interface on the front panel of the LABVIEW. This user interface makes the user freely to select the operating mode and adjust the normal temperature of the engine and the time of the engine starter motor.

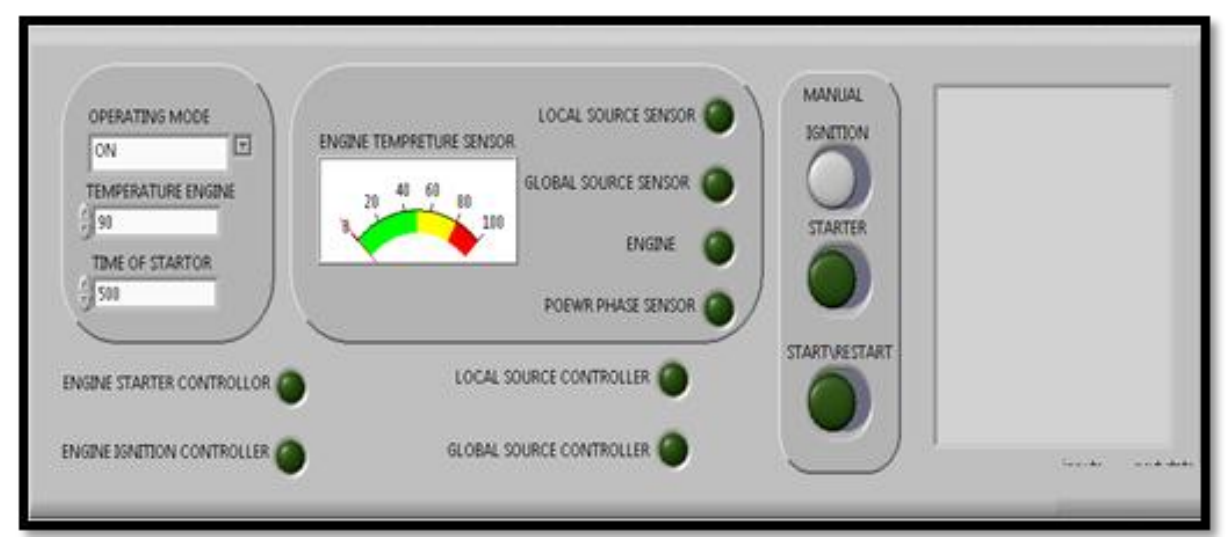

Figure 8. User interface of automatic power changer 


\subsection{Level controller system}

The main service of the fuel station is providing the fuel for the vehicles. A level controller system designed to protect the quality of the fuel and make the service provided by the fuel station better. The outputs of the level controller system are dispensing motor controller, submersible motor controller and filling motor controller. The data that entered into the controller are level sensor, density sensor, and water sensor data. Level sensor and density sensor replaced by a potentiometer to emulate its behavior. The anti oscillation technique used to prevent the oscillation of the lodes that depend on the level controller system. Figure 9 shows the user interface of the level controller system.

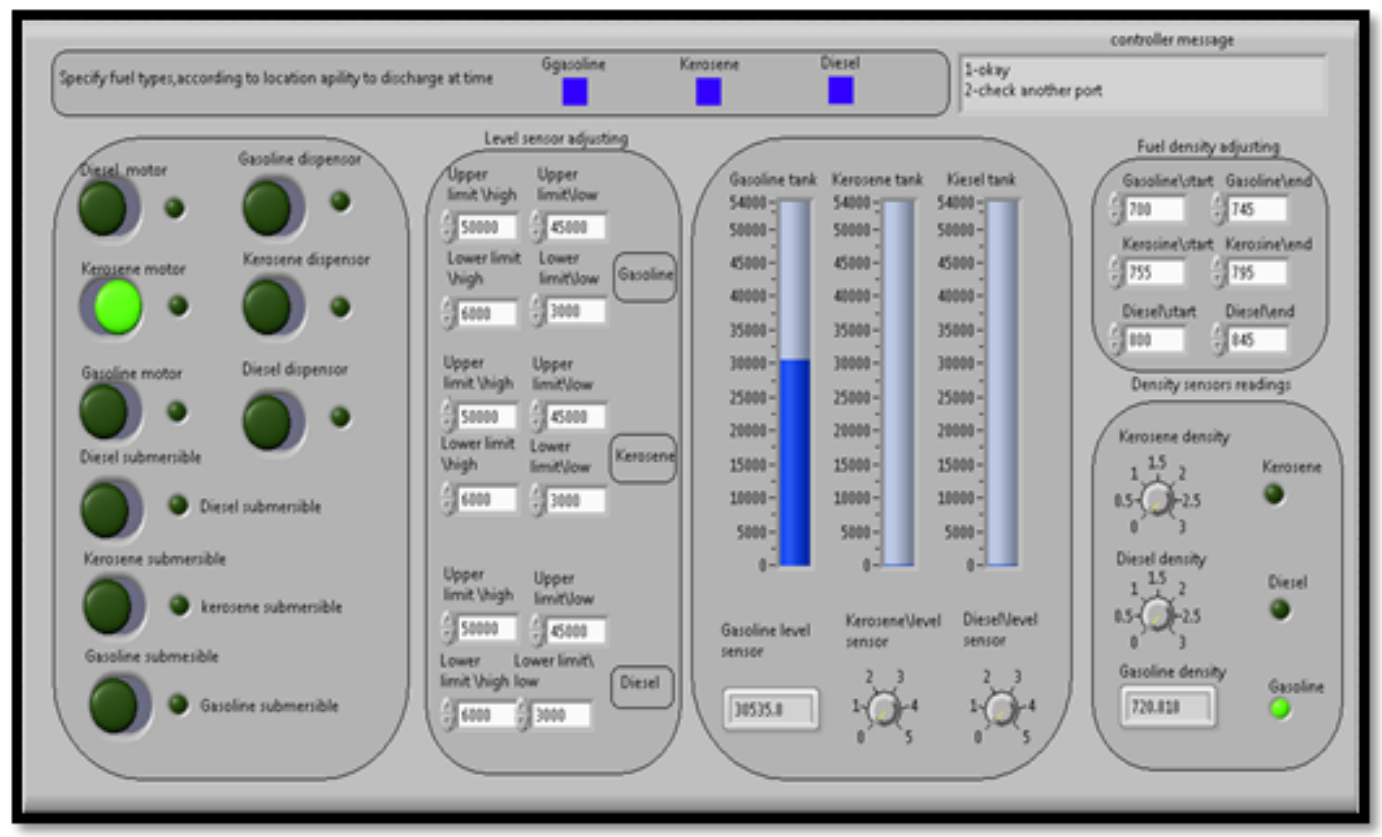

Figure 9. User interface of level and density controller system

\section{RESULTS}

The results of the subsystems which have affected on the same actuators make the results ambiguous for individual subsystem. The Activateldeactivate subsystems strategy used to solve this problem. The Activation used to the under test subsystem and deactivation for the others. Activation by varying the inputs of the system and get the results. Deactivation by making inputs in normal mode or eliminate system affect by jumping the subsystem alarm. The results of the main controller subsystems illustrated in Table 2, Table 3, Table 4, Table 5, and Table 6.

Table 2. The Results of Fire Protection Controller

\begin{tabular}{|c|c|c|c|c|c|c|c|c|}
\hline No. & $\begin{array}{c}\text { Smoke } \\
\text { Sensor }(0,1)\end{array}$ & $\begin{array}{l}\text { Temperature } \\
\text { Sensor }\left(\mathrm{C}^{\circ}\right)\end{array}$ & $\begin{array}{c}\text { Fire System } \\
\text { type }\end{array}$ & $\begin{array}{c}\text { Fire } \\
\text { Buzzer }\end{array}$ & $\begin{array}{l}\text { Fire Fighting } \\
\text { Controller }\end{array}$ & $\begin{array}{l}\text { Filling Motor } \\
\text { Controller }\end{array}$ & $\begin{array}{l}\text { Dispenser } \\
\text { Controller }\end{array}$ & $\begin{array}{c}\text { Submersible } \\
\text { Controller }\end{array}$ \\
\hline 1 & 0 & $<70$ & Compound & Off & Off & On & On & On \\
\hline 2 & 0 & $>70$ & Compound & On & On & Off & Off & Off \\
\hline 3 & 1 & $<70$ & Compound & On & On & Off & Off & Off \\
\hline 4 & 1 & $>70$ & Compound & On & On & Off & Off & Off \\
\hline 5 & 0 & $<70$ & Smoke sensor & Off & Off & On & On & On \\
\hline 6 & 0 & $>70$ & Smoke sensor & Off & Off & On & On & On \\
\hline 7 & 1 & $<70$ & Smoke sensor & On & On & Off & Off & Off \\
\hline 8 & 1 & $>70$ & Smoke sensor & On & On & Off & Off & Off \\
\hline 9 & 0 & $<70$ & $\begin{array}{l}\text { Temperature } \\
\text { sensor }\end{array}$ & Off & Off & On & On & On \\
\hline 10 & 0 & $>70$ & $\begin{array}{l}\text { Temperature } \\
\text { sensor }\end{array}$ & On & On & Off & Off & Off \\
\hline 11 & 1 & $<70$ & $\begin{array}{l}\text { Temperature } \\
\text { sensor }\end{array}$ & Off & Off & On & On & On \\
\hline 12 & 1 & $>70$ & $\begin{array}{c}\text { Temperature } \\
\text { sensor }\end{array}$ & On & On & Off & Off & Off \\
\hline
\end{tabular}


Table 3. The Results of Temperature Protection Controller

\begin{tabular}{|c|c|c|c|c|c|}
\hline \multirow{2}{*}{$\dot{z}$} & \multicolumn{2}{|c|}{$\begin{array}{l}\text { Temperature } \\
\text { Sensor }\left(C^{\circ}\right)\end{array}$} & \multirow{2}{*}{ 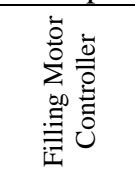 } & \multirow{2}{*}{ 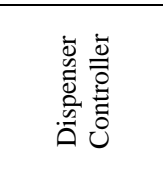 } & \multirow{2}{*}{ 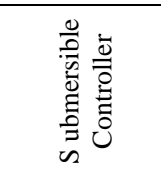 } \\
\hline & $\begin{array}{l}\text { Last } \\
\text { Case }\end{array}$ & $\begin{array}{l}\text { Present } \\
\text { Case }\end{array}$ & & & \\
\hline 1 & 1 & $5<\mathrm{TS}<45$ & $5<\mathrm{TS}<45$ & On & On \\
\hline 2 & 2 & $5<\mathrm{TS}<45$ & $45<\mathrm{TS}<50$ & On & On \\
\hline 3 & 3 & $5<\mathrm{TS}<45$ & $1<\mathrm{TS}<5$ & On & On \\
\hline 4 & 4 & $45<\mathrm{TS}<50$ & $45<\mathrm{TS}<50$ & Same last out & Same last out \\
\hline 5 & 5 & $45<\mathrm{TS}<50$ & $\mathrm{TS}>50$ & Off & Off \\
\hline 6 & 6 & $1<\mathrm{TS}<5$ & $1<\mathrm{TS}<5$ & Same last out & Same last out \\
\hline 7 & 7 & $1<\mathrm{TS}<5$ & $\mathrm{TS}<1$ & Off & Off \\
\hline 8 & 8 & $\mathrm{TS}>50$ & $\mathrm{TS}>50$ & Off & Off \\
\hline 9 & 9 & $\mathrm{TS}>50$ & $45<\mathrm{TS}<50$ & Off & Off \\
\hline 10 & 10 & $\mathrm{TS}<1$ & $1<\mathrm{TS}<5$ & Off & Off \\
\hline 11 & 11 & $\mathrm{TS}<1$ & $\mathrm{TS}<1$ & Off & Off \\
\hline
\end{tabular}

Table 4. The Results of Level Controller

\begin{tabular}{|c|c|c|c|c|c|c|}
\hline \multirow{2}{*}{ No } & \multicolumn{2}{|c|}{ Level controller } & \multirow{2}{*}{$\begin{array}{c}\text { Density } \\
\text { sensor }\end{array}$} & \multirow{2}{*}{$\begin{array}{l}\text { Dispenser } \\
\text { controller }\end{array}$} & \multirow{2}{*}{$\begin{array}{l}\text { Submersible } \\
\text { controller }\end{array}$} & \multirow{2}{*}{$\begin{array}{l}\text { Filling motor } \\
\text { controller }\end{array}$} \\
\hline & Last Case & Present Case & & & & \\
\hline 1 & $\mathrm{Y}<\mathrm{LS}<\mathrm{J}$ & $\mathrm{Y}<\mathrm{LS}<\mathrm{J}$ & 1 & On & On & On \\
\hline 2 & $\mathrm{Y}<\mathrm{LS}<\mathrm{J}$ & $\mathrm{J}<\mathrm{LS}<\mathrm{K}$ & 1 & On & On & On \\
\hline 3 & $\mathrm{Y}<\mathrm{LS}<\mathrm{J}$ & $\mathrm{X}<\mathrm{LS}<\mathrm{Y}$ & 1 & On & On & On \\
\hline 4 & $\mathrm{~J}<\mathrm{LS}<\mathrm{K}$ & $\mathrm{J}<\mathrm{LS}<\mathrm{K}$ & 1 & Same last out & Same last out & Same last out \\
\hline 5 & $\mathrm{~J}<\mathrm{LS}<\mathrm{K}$ & $\mathrm{LS}>\mathrm{K}$ & 1 & On & On & Off \\
\hline 6 & $\mathrm{X}<\mathrm{LS}<\mathrm{Y}$ & $\mathrm{X}<\mathrm{LS}<\mathrm{Y}$ & 1 & Same last out & Same last out & Same last out \\
\hline 7 & $\mathrm{X}<\mathrm{LS}<\mathrm{Y}$ & $\mathrm{LS}<\mathrm{X}$ & 1 & Off & Off & On \\
\hline 8 & $\mathrm{LS}>\mathrm{K}$ & $\mathrm{LS}>\mathrm{K}$ & 1 & On & On & Off \\
\hline 9 & $\mathrm{LS}>\mathrm{K}$ & $\mathrm{J}<\mathrm{LS}<\mathrm{K}$ & 1 & On & On & Off \\
\hline 10 & $\mathrm{LS}<\mathrm{X}$ & $\mathrm{X}<\mathrm{LS}<\mathrm{Y}$ & 1 & Off & Off & On \\
\hline 11 & $\mathrm{LS}<\mathrm{X}$ & $\mathrm{LS}<\mathrm{X}$ & 1 & Off & Off & On \\
\hline 12 & $\mathrm{Y}<\mathrm{LS}<\mathrm{J}$ & $\mathrm{Y}<\mathrm{LS}<\mathrm{J}$ & 0 & On & On & Off \\
\hline 13 & $\mathrm{Y}<\mathrm{LS}<\mathrm{J}$ & $\mathrm{J}<\mathrm{LS}<\mathrm{K}$ & 0 & On & On & Off \\
\hline 14 & $\mathrm{Y}<\mathrm{LS}<\mathrm{J}$ & $\mathrm{X}<\mathrm{LS}<\mathrm{Y}$ & 0 & On & On & Off \\
\hline 15 & $\mathrm{~J}<\mathrm{LS}<\mathrm{K}$ & $\mathrm{J}<\mathrm{LS}<\mathrm{K}$ & 0 & Same last out & Same last out & Off \\
\hline 16 & $\mathrm{~J}<\mathrm{LS}<\mathrm{K}$ & $\mathrm{LS}>\mathrm{K}$ & 0 & Off & Off & Off \\
\hline 17 & $\mathrm{X}<\mathrm{LS}<\mathrm{Y}$ & $\mathrm{X}<\mathrm{LS}<\mathrm{Y}$ & 0 & Same last out & Same last out & Off \\
\hline 18 & $\mathrm{X}<\mathrm{LS}<\mathrm{Y}$ & $\mathrm{LS}<\mathrm{X}$ & 0 & Off & Off & Off \\
\hline 19 & $\mathrm{LS}>\mathrm{K}$ & $\mathrm{LS}>\mathrm{K}$ & 0 & On & On & Off \\
\hline 20 & $\mathrm{LS}>\mathrm{K}$ & $\mathrm{J}<\mathrm{LS}<\mathrm{K}$ & 0 & On & On & Off \\
\hline 21 & $\mathrm{LS}<\mathrm{X}$ & $X<L S<Y$ & 0 & Off & Off & Off \\
\hline
\end{tabular}

Table 5. Automatic Power Changer Results Class A

\begin{tabular}{|c|c|c|c|c|c|c|c|c|c|}
\hline$\dot{0}$ & $\begin{array}{l}\dot{0} \\
0 \\
0 \\
0 \\
0 \\
0 \\
0 \\
0 \\
0 \\
\frac{\pi}{0} \\
\frac{0}{0} \\
0\end{array}$ & 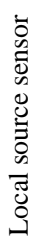 & 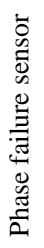 & 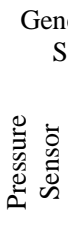 & 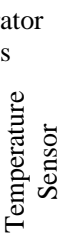 & 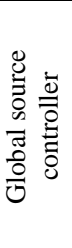 & 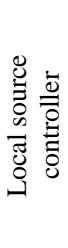 & $\begin{array}{l}\overline{0} \\
\overline{\bar{O}} \\
0 \\
0 \\
0 \\
0 \\
0 \\
.0 \\
.00 \\
0.0\end{array}$ & 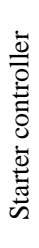 \\
\hline 1 & 1 & 0 & 0 & $\mathrm{~d}$ & $\mathrm{~d}$ & 0 & 0 & 1 & 1 \\
\hline 2 & 1 & $\mathrm{~d}$ & 1 & $\mathrm{~d}$ & d & 1 & 0 & 0 & 0 \\
\hline
\end{tabular}


Table 6. Automatic Power Changer Results Class B

\begin{tabular}{|c|c|c|c|c|c|c|c|c|c|c|}
\hline$\dot{z}$ & 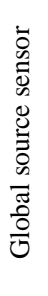 & 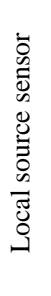 & 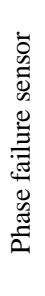 & 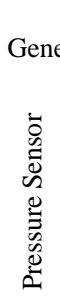 & 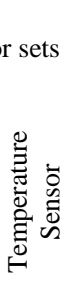 & 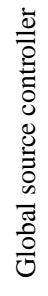 & 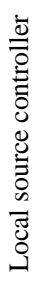 & 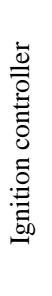 & 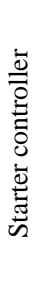 & 总 \\
\hline 1 & 1 & 0 & 0 & $\mathrm{~d}$ & $\mathrm{~d}$ & 0 & 0 & 1 & 1 & \\
\hline 2 & 0 & 0 & $\mathrm{~d}$ & d & $\mathrm{d}$ & 0 & 0 & 1 & 1 & Input States \\
\hline 3 & 0 & 0 & $\mathrm{~d}$ & d & $\mathrm{d}$ & 0 & 0 & 1 & 1 & $\begin{array}{l}\text { Second } \\
\text { Attempt }\end{array}$ \\
\hline 4 & 0 & 0 & $\mathrm{~d}$ & d & $\mathrm{d}$ & 0 & 0 & 1 & 1 & Third Attempt \\
\hline 5 & 0 & 1 & 1 & 1 & $<90$ & 0 & 1 & 1 & 0 & $\begin{array}{l}\text { Normal Local } \\
\text { Source }\end{array}$ \\
\hline 6 & 0 & 1 & 1 & d & $>90$ & 0 & 0 & 0 & 0 & $\begin{array}{c}\text { High } \\
\text { temperature }\end{array}$ \\
\hline 7 & 0 & 1 & 1 & 0 & $\mathrm{~d}$ & 0 & 0 & 0 & 0 & $\begin{array}{l}\text { Low Oil } \\
\text { Pressure }\end{array}$ \\
\hline 8 & 0 & 1 & 0 & $d$ & d & 0 & 0 & 0 & 0 & \\
\hline 9 & 0 & 0 & $\mathrm{~d}$ & $\mathrm{~d}$ & d & 0 & 0 & 0 & 0 & $\begin{array}{l}\text { The Engine } \\
\text { Fails to Run }\end{array}$ \\
\hline
\end{tabular}

\section{RESULTS DISCUSSIONS}

The results of the fire protection controller presented in Table 2, where jump fire protection button deactivated. When jump fire button activated the system don't work and still without action. The buttons above reached through the user interface of the fire protection system shown in the Figure 4. Also the smoke sensor, temperature sensor, fire protection type, fire buzzer, and actuator controllers that found on top of the Table 2 are appearing in the user interface of the fire protection controller. The combination of the user interface with the results table gives a good view of the fire protection performance.The results of the normal temperature controller are shown in Table 3 , where jump temperature protection button deactivated. When jump temperature protection button activated the system don't work and still without action. The level controller results are shown in Table 4. The results depend on the level sensor (variable resistance), density sensor (variable resistance), and water sensor. The outputs of the controller concern with dispenser, submersible, and filling motor controllers. Although the conditions of work needed for dispenser and submersible same, but there are inherent dependencies of the submersible on dispensers, where submersible don't run else at least one dispenser in on mode. In case of water sensor detect water in fuel tank, the outputs of controller turn off the filling, dispensing, and submersible motor and an alarm of high (unaccepted water content) water content in the fuel tank. In the density sensor field the " 1 ".

Mean the density of fuel in the filing system in the pre specified range of fuel type. The variables $(\mathrm{X}, \mathrm{Y}, \mathrm{J}, \mathrm{K})$ represent the level limits upper and lower limits which specified by a user through the user interface in Figure 8. This limit used to create guides between operating regions of the anti oscillation controller shown in Figure 5. The results of the automatic power changer are shown in classes as in Tables 5, and 6. The results are on (Automatic) mode only where there are three another results come from another three modes of operation. The modes else on mode are off, local source only, and global source only. The "off" mode makes outs of the controller in the "off" state. The local source only mode makes the global source controller in the "off" state and manually operation of generator set (local source). The global source only mode makes the local source controller in the "off" state and global source controller in the "on" state. The on mode is the automatic mode, which changes the power source according to the presence or absence of the global source power. Table 5 shows the results under presence of global power source and normal and abnormal phase voltage quality. The letter "d" referee to don't care input.

Table 6 shows the results under the absence of the global power source of the No. " 1 " in the Table 6, where the controller tries to turn on the local generator set then check the presence of the local power and engine sensors. Maximum number of turns on trying is three consequent attempts. The letter "d" referee to don't care input.

\section{CONCLUSIONS}

This paper demonstrates to an automation and monitoring controller, which used NI WSN and Arduino microcontroller. The controller, which developed by LABVIEW environment tested and give good 
results which encourage the researcher to imply the developed code for the NI reconfigure able input output device which make the controller applicable to real-time application.

\section{REFERENCES}

[1] Waqas Ikram and Nina F. Thornhill, "Wireless Communication in Process Automation: A Survey of Opportunities, Requirements, and Challenges", UKACC International Conference on Control, UK, September 2010.

[2] Gang Zhao and Davis, "Wireless Sensor Networks for Industrial Process Monitoring and Control: A Survey", Microthink Institute, Network Protocols and Algorithms, vol. 3, no. 1, pp. 46-63, 2011.

[3] P. Velmani, "Survey on Applications and Challenges of Wireless Sensor Network in Industries", International Journal of Computer Science and Communication Network, vol. 4, no. 2, pp. 41-45, 2013.

[4] A. Ajith Kumar S., Knut Øvsthus, Lars M. Kristensen, "An Industrial Perspective on Wireless Sensor Networks A Survey of Requirements, Protocols, and Challenges", IEEE Communication Surveys and Tutorials, vol. 16, no. 3, pp. 1391-1412, 2014.

[5] Basil Hamed, "Design and Implementation of Smart House Control Using LABVIEW", International Journal of Soft Computing and Engineering (IJSCE), vol. 1, no. 6, pp. 98-106, 2012.

[6] S. Rajesh Kumar and S. Rameshkumar, "Industrial Temperature Monitoring and Control System through Ethernet LAN", International Journal of Engineering and Computer Science, vol. 2, no. 6, pp. 1988-1991, 2013.

[7] S. Sathiysmoorthy, "Industrial Application of Machine Vision", International Journal of Research in Engineering and Technology (IJRET), vol. 3, no. 7, pp. 678-682, 2014.

[8] Poornendu K, Manoj G and Kannan E. P., "Data Acquisition and Controlling in Thermal Power Plants Using a Wireless Sensor Network and LABVIEW”, International Journal of Engineering \& Technology (IJERT), vol. 4, no. 7, pp. 568-572, 2015.

[9] Arti Dhadiwiwal, Payal Disoja and Prof Amit H. Palve, "Smart Centralized Power Control for Buildings Using WSN", Green Computer and Internet of Things (ICGCoT), IEEE International Conference, 2015.

[10] "LM35 Precision Centigrade Temperature Sensor", 2000, available at, http://www.ece.usu.edu/ecestore/spec/lm35dt-3p.pdf. Accessed on June 2017.

[11] Technical Data of MQ-2 Gas Sensor", available at, http://www.haoyuelectronics.com/Attachment/MQ-2/MQ2.pdf. Accessed on June 2017

\section{BIOGRAPHIES OF AUTHORS}

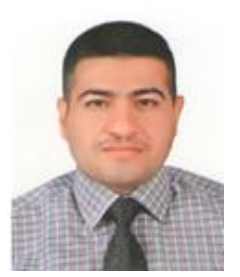

Ehab Abdul Razzaq Hussein, PhD. MSc. Electrical Engineering was born in Babylon on January 1, 1976. He obtained his BSc degree (1997) in Electrical Engineering at the Faculty of Engineering, University of Babylon and MSc degree (2000), in electrical engineering at the Department of Electrical Engineering, University of Technology and his PhD. Degree from the Department of Electrical Engineering at the Faculty of Engineering, University of Basrah, Currently he works as assistant professor at the Electrical Department at the Faculty of Engineering, University of Babylon. His main interest is signal processing, analysis, information transition, sensors and control system analysis.

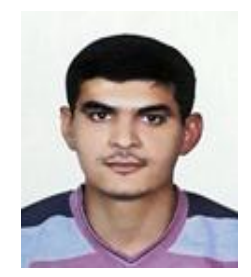

Mahran Obaid Waheed, Electrical Engineer was born in Babylon, 1982. He obtained his BCs degree (2005) in Electrical Engineering at the Faculty of Engineering, University of Babylon. Currently he is studying for a master's degree in Electrical Engineering at the Faculty of Engineering, University of Babylon. His main interest is signal processing, analysis, measurement and calibration unit and control system analysis. 